\title{
SEQUÍA: DEFINICIONES, TIPOLOGÍAS Y MÉTODOS DE CUANTIFICACIÓN
}

\author{
Óscar Marcos Valiente \\ Departamento de Geografía Física y Análisis Geográfico Regional \\ Universidad de Barcelona
}

\section{RESUMEN}

La definición de sequía ha sido el objeto de numerosos estudios científicos, pero la diversidad de tipologías climáticas existentes sobre el planeta hace casi imposible utilizar el mismo umbral de déficit pluviométrico en dos lugares diferentes. En consecuencia, se establecen numerosas definiciones de sequía que dificultan la comparación de magnitudes entre áreas geográficas, y se emplean herramientas de cuantificación de los eventos secos adaptadas a las necesidades de cada tipología climática. El artículo presenta las diversas herramientas metodológicas creadas para cuantificar los diferentes tipos de sequía existentes: meteorológica, agrícola, hidrológica y socioeconómica.

Palabras clave: sequía, métodos cuantitativos, definición de sequía, sequía meteorológica, sequía agrícola, sequía hidrológica, sequía socioeconómica.

\begin{abstract}
Drought definition has been the object of several scientific studies, but the diversity of climatic typologies troughout the planet difficults the use of the same rainfall deficit threshold in two different places. As a result, a huge number of drought definitions are established, increasing the difficulties to compare magnitudes among geographic areas, and drought quantification tools are adapted to the needs of each climate type. The article presents the diverse methodological tools created to quantify drought episodes, distinguishing between the different types of drought: meteorological, agricultural, hydrological and socioeconomic.
\end{abstract}

Key words: Drought, quantitative methods, drought definition, meteorological drought, agricultural drought, hydrological drought, socio-economic drought.

\footnotetext{
* Este trabajo forma parte de una tesis de doctorado titulada «Evaluación de la vulnerabilidad a la sequía en el Nordeste de Brasil mediante indicadores socioclimáticos», dirigida por el Dr. José Luis Luzón Benedicto.
} 


\section{Definición de sequía}

La definición de sequía puede parecer sencilla, y si consultamos un buen número de diccionarios o enciclopedias, encontraremos que en casi todos ellos se nos dice algo parecido a «deficiencia de precipitaciones durante un período de tiempo relativamente prolongado».

Ahora bien, ahí terminan las coincidencias, puesto que la cuantificación de la deficiencia y el tiempo expresados en la definición varía notablemente según el espacio geográfico sobre el que deba aplicarse. A este tipo de definiciones enciclopédicas, que no establecen umbrales de referencia y no tienen en cuenta la realidad climática del área afectada por el déficit pluviométrico, se les denomina conceptuales.

Cuando la definición precisa el momento de inicio, finalización e intensidad de la sequía, hablamos de definiciones operacionales, las cuales requieren un conocimiento más detallado de las variables climáticas, así como disponer de series de datos de diferente escala, según el nivel de detalle deseado (horario, diario, mensual, estacional, anual, decenal...).

Wilhite y Glantz (1985) detectaron más de 150 definiciones de este tipo, categorizándolas en cuatro grupos según la disciplina científica desde la que sea analizado el fenómeno: sequía meteorológica, sequía hidrológica, sequía agrícola y sequía socioeconómica. Existen clasificaciones más simples, como la de SUDENE (1999), que prescinde del tipo meteorológico y divide las sequías en hidrológicas, agrícolas y efectivas (estas últimas equivalentes a socioeconómicas), y más detalladas, como la de Subrahmanyam (1967), que distingue hasta seis tipos de sequía (meteorológica, climática, atmosférica, agrícola, hidrológica y de gestión hídrica). Sin embargo, ninguna de ellas aporta información conceptualmente mejor sustentada que la propuesta por Wilhite y Glantz (1985).

\section{Sequía meteorológica}

Basada en datos climáticos, es una expresión de la desviación de la precipitación respecto a la media durante un período de tiempo determinado. Ante la dificultad de establecer una duración y magnitud del déficit pluviométrico válidas para diferentes áreas geográficas, algunas definiciones de sequía optan por no especificar umbrales fijos.

Por ejemplo, Palmer (1965) define sequía meteorológica como el «intervalo de tiempo, generalmente con una duración del orden de meses o años, durante el cual el aporte de humedad en un determinado lugar cae consistentemente por debajo de lo climatológicamente esperado o del aporte de humedad climatológicamente apropiado», mientras que Russell et al. (1970) son más concisos: «Falta prolongada de precipitación, inferior a la media».

En la mayoría de casos, las definiciones de sequía meteorológica presentan información específica para cada región particular, que varía en función de las características del clima regional. Por tanto, es imposible extrapolar una definición de una región a otra:

- INDONESIA (BALI): «Período de seis días sin lluvias» (Hudson y Hazen, 1964).

- ESPAÑA: «En las diferentes cuencas hidrográficas españolas pueden considerarse años secos aquellos cuya precipitación experimenta la siguiente reducción respecto a la media anual: Cantábrico, Duero y Ebro, 15-25\%; Guadalquivir, 20-25\%; Guadiana/Tajo, 30\%; Levante y Sureste, 40-50\%» (Olcina, 1994). 
- GRAN BRETAÑA: «Período de al menos quince días consecutivos con precipitación diaria inferior a 0,25 mm» (Goudie, 1985).

- INDIA: «Situación en la que la precipitación estacional anual es deficiente en, al menos, dos veces la desviación típica» (Ramdas, 1960).

- INDIA (Indian Meteorological Office): «La precipitación es inferior al $80 \%$ de los niveles normales» (Dhar et al., 1979).

- LIBIA: «Precipitación anual inferior a 180 mm» (Hudson y Hazen, 1964).

- NORDESTE DE BRASIL: «Precipitaciones mensuales durante el período lluvioso inferiores a $100 \mathrm{~mm}$, o inferiores al $80 \%$ de la media a lo largo de 90 días, durante los cuales se producen intervalos superiores a 10 días con valores inferiores a 10 $m m »$ (SUDENE, 1981).

- URSS: «Período de 10 días con una lluvia total que no excede 5 mm» (Krishnan, 1979).

Algunas propuestas no sólo contemplan la precipitación, sino que añaden la incidencia de la evapotranspiración, con lo cual crean confusión con la definición de déficit hídrico, que forma parte del ciclo climático anual y no tiene por qué constituir una situación de sequía.

Tal es el caso de Rind et al. (1990), que definen sequía meteorológica como «la condición resultante de un exceso de demanda atmosférica de humedad respecto a su aporte $(E T P>P) \gg$.

\section{Sequía agrícola}

Por ser el primer sector económico que resulta afectado por la escasez de precipitaciones, la agricultura adquiere una especial relevancia en relación con la sequía. Así, se produce una sequía agrícola cuando no hay suficiente humedad en el suelo para permitir el desarrollo de un determinado cultivo en cualquiera de sus fases de crecimiento.

Dado que la cantidad de agua es diferente para cada cultivo, e incluso puede variar a lo largo del crecimiento de una misma planta, no es posible establecer umbrales de sequía agrícola válidos ni tan siquiera para una única área geográfica. Aún así, Kulik (1962) se arriesga a ello, definiendo sequía agrícola como el «período durante el cual sólo hay 19 mm de agua disponible en los primeros $20 \mathrm{~cm}$ de suelo».

Este tipo de sequía, por depender no sólo de las condiciones meteorológicas, sino también de las características biológicas del cultivo y las propiedades del suelo, no es equivalente a la sequía meteorológica. Si los niveles de humedad en el subsuelo son suficientes para proporcionar agua a un determinado tipo de cultivo durante el período que dure la sequía meteorológica, no llegará a producirse una sequía agrícola.

\section{Sequía hidrológica}

Hace referencia a una deficiencia en el caudal o volumen de aguas superficiales o subterráneas (ríos, embalses, lagos, etc.). Al producirse un desfase entre la escasez de lluvias o nieves y la reducción del caudal de ríos o el nivel de lagos y embalses, las mediciones hidrológicas no pueden ser utilizadas como un indicador del inicio de la sequía, pero sí de su intensidad. 


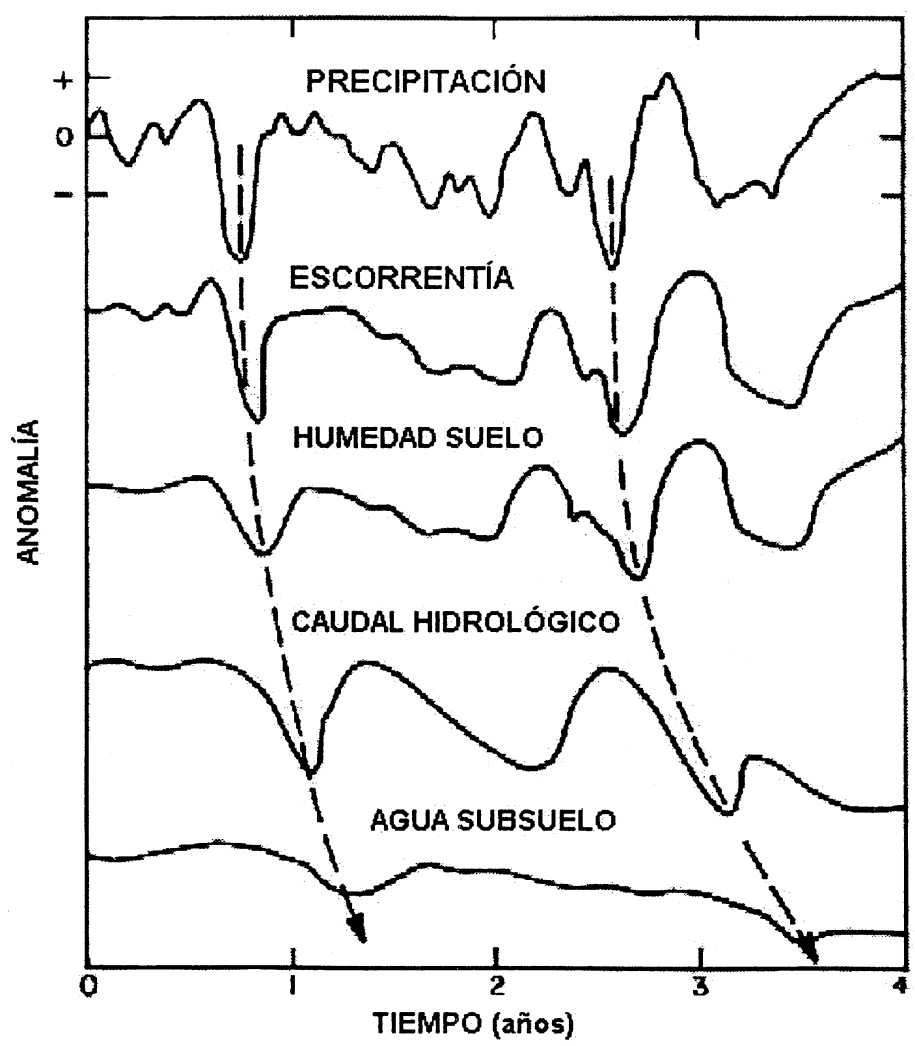

FUENTE: Entekhabi et al. (1992).

FIGURA 1. Desfase temporal en la propagación de anomalías pluviométricas hacia los diversos niveles del ciclo hidrológico.

A diferencia de la sequía agrícola, que tiene lugar poco tiempo después de la meteorológica, la sequía hidrológica puede demorarse durante meses desde el inicio de la escasez pluviométrica (véase la figura $\mathrm{n}^{\mathrm{o}} 1$ ) o, si las lluvias retornan en poco tiempo, no llegar a manifestarse. Por tanto, la secuencia temporal es: sequía meteorológica, a continuación sequía agrícola y, en último lugar, sequía hidrológica.

La capacidad de gestionar los recursos hídricos hace que la sequía hidrológica no dependa exclusivamente del volumen de agua existente en los depósitos naturales o artificiales, sino que también es determinante la forma en que se emplea el agua embalsada. Linsley et al. (1975) toman en consideración estos aspectos, al definir sequía hidrológica como el «período durante el cual los caudales son inadecuados para satisfacer los usos establecidos bajo un determinado sistema de gestión de aguas». 


\section{Sequía socioeconómica}

Se produce cuando la disponibilidad de agua disminuye hasta el punto de producir daños (económicos o personales) a la población de la zona afectada por la escasez de lluvias. Para hablar de sequía socioeconómica no es necesario que se produzca una restricción del suministro de agua, sino que basta con que algún sector económico se vea afectado por la escasez hídrica. La pujante presión antrópica sobre el recurso agua hace que cada vez sea mayor la incidencia de la sequía socioeconómica, con pérdidas económicas crecientes, incluso en el caso de una sequía meteorológica leve.

En términos generales, la sequía socioeconómica sucede a la agrícola, pero en regiones menos desarrolladas, donde este sector tiene un mayor peso en la economía, y su incidencia es más directa e inmediata que en regiones desarrolladas, la sequía agrícola constituye en sí misma el inicio de la sequía socioeconómica.

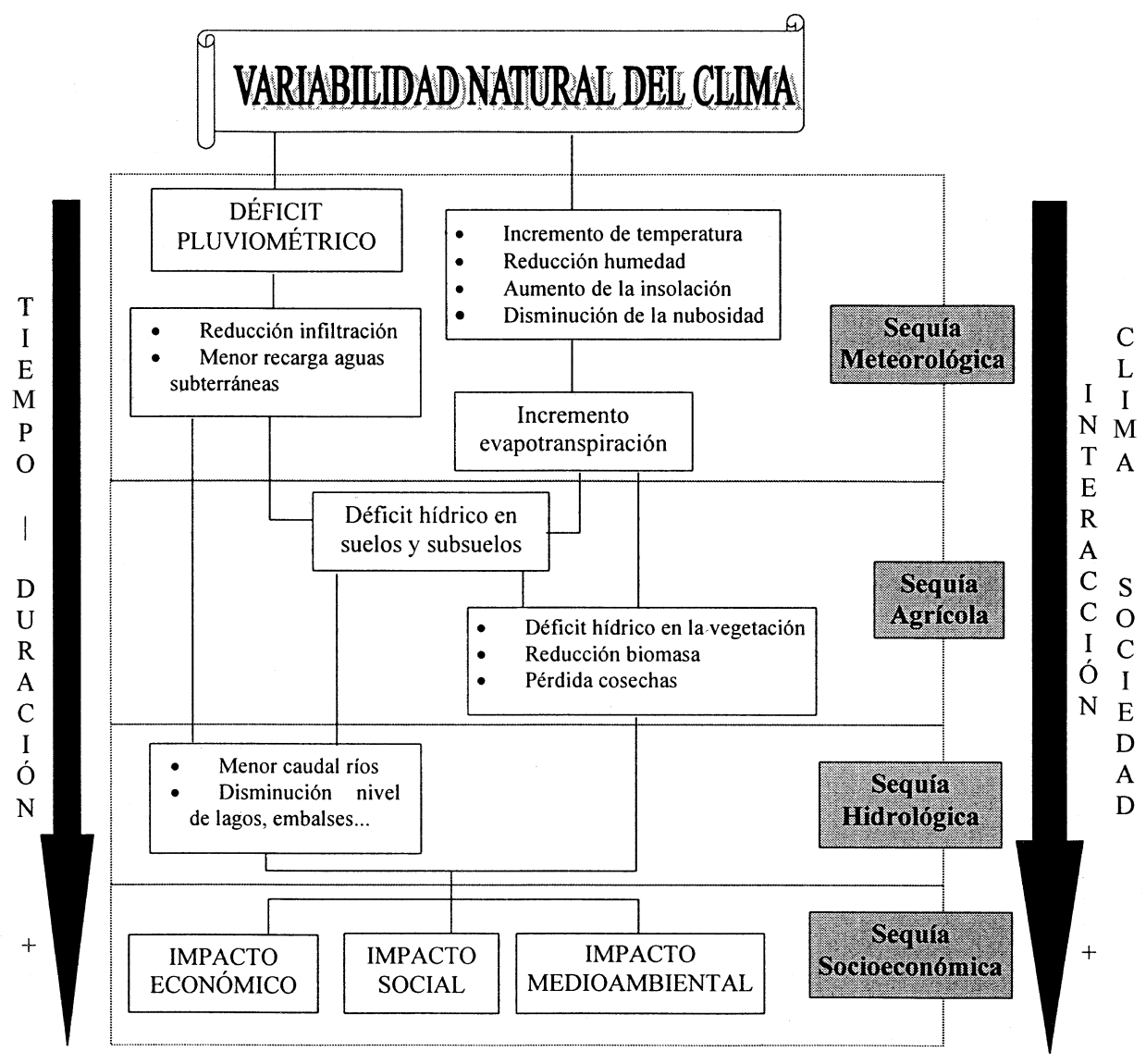

FUENTE: Basado en NDMC (1995).

FiguRa 2. Secuencia de evolución de los diversos tipos de sequía operacional. 
En el caso de la sequía socioeconómica, son tan determinantes la magnitud y la duración del evento seco como la forma en que la economía y la sociedad desarrollan sus actividades, ya que determinadas acciones incrementan la vulnerabilidad a la escasez de precipitaciones.

En función de esa vulnerabilidad, los efectos de la sequía sobre la economía y la sociedad, medidos en forma de pérdidas materiales, población afectada o pérdida de vidas humanas, adquirirán mayor o menor relevancia, llegando a configurar situaciones en las que la sequía es considerada una catástrofe.

Las diferentes formas en que la sociedad y la economía de una región pueden verse afectadas por la escasez de precipitaciones quedan reflejadas en las diversas definiciones existentes de este tipo de sequía:

- «Escasez de precipitaciones tan cuantiosa y continuada como para afectar dañinamente a la vida animal y vegetal de un lugar, y para reducir las reservas de agua para usos domésticos y para el funcionamiento de plantas energéticas, especialmente en aquellas regiones donde la lluvia es normalmente suficiente para tales necesidades» (Havens, 1954).

- «Desvío negativo extenso y significativo de la precipitación con relación al régimen alrededor del cual una sociedad se ha establecido» (Rasmussen, 1987).

- «Fenómeno accidental de duración variable, marcado por un déficit anormal de agua, que produce una ruptura del equilibrio natural, una desorganización de la gestión del país, una reducción considerable de la producción, y conflictos sociales que pueden ser irreversibles. Se produce en todos los dominios climáticos, pero afecta más duramente a las regiones semiáridas marcadas por una fuerte variación interanual de las lluvias» (Toupet, 1989).

- «Déficit hídrico inusual que genera impactos adversos en la sociedad que la padece, alterando el normal desenvolvimiento de su vida colectiva» (Pita López, 1995).

- «Episodio de baja precipitación y mala distribución de las lluvias, que hace difícil la alimentación de la población y los rebaños, e imposibilita el mantenimiento de las reservas de agua necesarias para el consumo humano y animal» (SUDENE, 1999).

Los cuatro tipos de evento seco analizados reflejan un proceso de evolución de la sequía, determinado tanto por la variabilidad natural del clima, como por la duración del evento, y por la interacción entre clima y sociedad (véase la figura $\mathrm{n}^{\circ} 2$ ). Cada tipo de sequía es cuantificado para poder establecer la magnitud y duración del período de escasez pluviométrica, pero la diversidad de definiciones existentes determina la existencia de numerosos métodos de cuantificación, tal y como se puede observar en el siguiente apartado.

\section{Métodos cuantitativos de caracterización de la sequía}

Las definiciones operativas de los diversos tipos de sequía presentadas en el apartado anterior necesitan ser trasladadas a un formato numérico para poder precisar las características de los eventos secos. Ahora bien, ¿qué componentes nos permiten definir una sequía?

Desde el punto de vista antrópico, que es el que determina que un evento extremo de la naturaleza de los episodios de escasez pluviométrica se convierta en un riesgo natural, 
Burton et al. (1978) definen siete parámetros con los que caracterizarla, uno independiente (magnitud), cuatro correspondientes al componente temporal (duración, frecuencia, velocidad de implantación y espaciamiento temporal), y dos al espacial (extensión y dispersión espacial). Para cuantificar estos parámetros no es suficiente con medir el número de días de lluvia, o expresar percepciones en referencia a la escasez de precipitaciones, sino que es necesario aplicar cálculos matemáticos con los que establecer detalladamente en qué medida ha habido déficit pluviométrico y cuáles son los límites temporales del episodio seco.

En referencia a la sequía meteorológica e hidrológica (según los trabajos de Dracup et al. (1980), y Martín-Vide (1998), respectivamente), cada parámetro puede definirse y ser medido de forma individualizada:

- La magnitud es el déficit medio de precipitación o caudal durante el periodo de duración del evento seco. Puede medirse como la anomalía (en porcentaje) o la diferencia (en valor absoluto) de precipitación o caudal para el período seleccionado. Dracup et al. (1980) señalan que, además de la magnitud, puede delimitarse la severidad de una sequía, definida como el déficit acumulado de caudal o precipitación para la duración del período seco.

- La duración es el tiempo (número total de días, meses o años consecutivos) durante el cual la precipitación o el caudal totales registrados son inferiores a la precipitación o el caudal medio de ese mismo período. Dracup et al. (1980) relacionan los parámetros magnitud, severidad y duración, de tal forma que partiendo de la fórmula MAGNITUD = SEVERIDAD / DURACIÓN, cualquiera de los tres puede ser conocido.

- La frecuencia es el número de casos que se producen durante un período determinado. Puede medirse también a través de la probabilidad empírica de que la precipitación o el caudal sean inferiores a la media, o del período de retorno del evento seco (intervalo medio de tiempo dentro del que puede esperarse que ocurra una sequía de determinada magnitud).

- La velocidad de implantación es el tiempo transcurrido entre el momento de inicio del déficit de precipitación o caudal, y el momento en que ese déficit alcanza su valor máximo.

- El espaciamiento temporal es el tiempo transcurrido entre diversos períodos secos, lo que nos proporciona una medida de la regularidad o aleatoriedad del fenómeno. Mediante este parámetro se obtiene una primera aproximación a la previsibilidad de la sequía.

- La extensión es la superficie total en la que se registra déficit hídrico.

- La dispersión espacial es la medida del grado de difusión o concentración de la anomalía de caudal o de precipitaciones.

En función de los extremos relativos que pueden asignarse a los parámetros espaciales y temporales, Burton et al. (1978) definen la sequía como un riesgo penetrante, dada su frecuencia intermedia, su larga duración, su lenta velocidad de ataque, su relativamente aleatorio espaciamiento temporal, su gran extensión y su elevada difusión espacial. Se trata, por tanto, de un evento extremo totalmente contrapuesto al riesgo intensivo, ejemplificado por el terremoto, que es poco frecuente, de corta duración, espacialmente concentrada, con una extensión limitada, una rápida velocidad de ataque y un espaciamiento temporal relativamente aleatorio. 
Prescindiendo de las unidades de medida detalladas para cada parámetro en su definición, existe una amplia variedad de índices y ecuaciones dedicadas a caracterizar los eventos secos en sus dos parámetros más relevantes: magnitud (o severidad) y duración. Sobre la bondad y los defectos de los diversos métodos de cuantificación de las sequías se han escrito múltiples artículos (Bowles et al., 1980; Alley, 1984; Karl, 1986; Soule, 1992; Meyer y Hubbard, 1995; NDMC, 1996; Meshcherskaya y Blazhevich, 1997; Byun y Wilhite, 1999; NDMC, 1999).

Existen índices complejos, como el PDSI de Palmer, y otros simples como el cálculo del porcentaje de precipitación respecto a la media para una determinada escala temporal. Pese a que todos estos métodos de cuantificación han sido creados con voluntad universal, algunos no son aplicables en determinadas zonas del planeta, como los elaborados por W.C. Palmer. A continuación se presentan una recopilación de los índices más importantes, detallando sus principales características, y clasificados según el tipo de sequía al que son aplicables (meteorológica, hidrológica y agrícola). En el caso de que un mismo índice sea aplicable a más de un tipo de sequía, se asigna a aquél en el cual haya sido utilizado con más asiduidad.

\section{Métodos de cuantificación de la sequía meteorológica}

\subsection{Porcentaje de la precipitación media}

Este cálculo simple se expresa mediante el cociente de la precipitación total registrada en un período determinado (mes, trimestre, estación, semestre, año, etc.) y la precipitación media (habitualmente por un período de 30 años) del mismo período de referencia, expresado el resultado en porcentaje. Pese a su simplicidad, es un método susceptible de equívocos, puesto que la precipitación media es un número abstracto que no tiene por qué ser equivalente a la mediana, que sí representa el valor o intervalo de mayor frecuencia de la serie. Además, presenta la dificultad de establecer los límites a partir de los cuáles se establece una situación de sequía, así como su severidad, puesto que el porcentaje de desviación escogido para un lugar o región no es aplicable a otra zona.

Pese a sus carencias, sigue siendo el índice de sequía más utilizado, ya que puede ser calculado incluso para aquellas zonas con poca disponibilidad de información climatológica. Bhalme y Mooly (1980) rebautizaron este método con las siglas BMDI (Bhalme \& Mooly Drought Index), pese a ser un procedimiento estadístico utilizado desde mucho antes.

\subsection{Cuantiles (quintiles, deciles y percentiles)}

Para evitar las limitaciones derivadas del anterior método, el establecimiento de los umbrales puede realizarse mediante el cálculo de quintiles, deciles y percentiles. La técnica consiste en dividir la distribución de ocurrencias pluviométricas durante un período temporal suficientemente largo, en intervalos para cada 20\% (quintil), 10\% (decil), o 1\% (percentil) de la distribución. Al ser valores totalmente referenciados en la serie pluviométrica, ofrecen unos límites más cercanos a la realidad climática del área estudiada. Para que el cálculo de este índice ofrezca resultados fiables y estadísticamente significativos, es necesario que la serie pluviométrica sea larga (como mínimo treinta años y, si es posible, más de cincuenta).

Los límites de referencia más utilizados son los correspondientes a los quintiles, de tal forma que un período es muy seco cuando el valor de precipitación total se sitúa entre el $20 \%$ de los valores más bajos, seco cuando se encuentra en el tramo del siguiente $20 \%$ de 
valores, normal cuando se sitúa en el tramo del $20 \%$ de valores intermedios, húmedo en el siguiente tramo del $20 \%$, y muy húmedo en el intervalo del $20 \%$ de los valores más elevados de la serie.

En caso de requerir una mayor precisión en los umbrales, puede prescindirse de los quintiles y recurrir a los deciles o percentiles para incrementar el número de intervalos. La escala de deciles establece diez intervalos para la serie de valores, cada uno de los cuales se reparte un $10 \%$ de los valores: sequía muy fuerte (extrema), sequía fuerte (severa), sequía moderada, sequía leve, sequía incipiente, humedad incipiente, humedad leve, humedad moderada, humedad fuerte (severa) y humedad muy fuerte (extrema).

Por ejemplo, el método de los deciles es utilizado por el servicio de vigilancia de sequías de Australia para medir la sequía meteorológica y poner en marcha los planes de intervención (Gibbs y Maher, 1967; Smith et al., 1993).

\subsection{Desviación estandarizada de precipitación}

Este índice se calcula restando a la precipitación total de un determinado período la media aritmética de ese período y dividiendo el resultado por la desviación típica. El resultado será próximo a cero para valores cercanos a la media, e igual a uno cuando la diferencia entre la lluvia registrada y la media del período sea próxima al valor de desviación típica. La estandarización de los valores permite que los resultados presenten una distribución normal. Aunque este método permite establecer comparaciones entre todo tipo de climas, presenta problemas en aquellas regiones con una elevada variabilidad pluviométrica, de tal forma que si la media aritmética es inferior a la desviación típica, el índice nunca podrá alcanzar el valor -1 . Tal es el caso en los valores anuales de regiones desérticas como la de Atacama, en Chile, donde un año con precipitación igual a cero, presentará una desviación estandarizada de precipitación mínima: entre -0,3 y -0,8. En ámbitos semiáridos, como el Nordeste de Brasil o el Norte Chico de Chile, algunas estaciones apenas alcanzan un valor mínimo de $-1,5$, por lo que el resultado no tendrá validez comparativa con otras regiones.

McKee et al. (1993), que desarrollaron una variante de esta metodología, denominándola Índice Estandarizado de Precipitación (SPI, Standarized Precipitation Index), fijan el inicio de un episodio de sequía en el caso de que el SPI sea continuamente negativo con un valor inferior o igual a -1,0, y su finalización en el momento en que el SPI adquiera valor positivo. Siguiendo con esta delimitación arbitraria, escogida para su aplicación en Colorado (Estados Unidos), cuando el SPI supera el umbral de $-1,0$ se habla de sequía moderada, si es inferior a -1,5 sequía severa, y para valores iguales o menores que -2,0 sequía extrema. Según estas determinaciones, en las áreas desérticas antes mencionadas no se producen episodios de sequía, puesto que el SPI nunca puede ser igual o inferior a -1,0, mientras que en algunas zonas semiáridas la sequía no podría alcanzar el nivel extremo. Los mismos autores definen el concepto de Magnitud de Sequía como la suma del SPI para todos los meses incluidos en el período de sequía, obteniendo una medida de la magnitud acumulada del evento seco.

\section{4. Índice de Severidad de la Sequía de Palmer (PDSI, Palmer Drought Severity Index)}

El PDSI fue creado por Palmer (1965) para medir las condiciones de humedad. El índice está basado en el balance hídrico, de tal forma que utiliza numerosas variables para reflejar el aporte y la demanda de agua. Por su formulación, el PDSI permite identificar 
adecuadamente la sequía meteorológica, responde lentamente a la agrícola y no detecta la hidrológica. El índice es utilizado para definir la intensidad de la sequía, pero el hecho de que en su concepción y en el establecimiento de los límites se basara en las condiciones climáticas de Iowa y Kansas hace que su aplicación se haya limitado a Estados Unidos, donde existen series mensuales del PDSI desde 1895.

Según el análisis crítico del índice realizado por Alley (1984) y Karl (1986), el PDSI destaca por su capacidad de medir la sequía con perspectiva histórica y posibilitar la realización de representaciones espaciales y temporales de sequías históricas. En cuanto a los aspectos negativos, son numerosas las limitaciones, incorrecciones y asunciones del PDSI, la mayoría de ellas relacionadas con el cálculo del balance hídrico y con el período utilizado para el cálculo de los valores medios en las fórmulas (1931-1960, que fue anormalmente cálido y seco en la mayor parte de Estados Unidos).

El PDSI ha sido escasamente utilizado fuera de Estados Unidos, y ofrece resultados poco satisfactorios en regiones donde la variabilidad pluviométrica es elevada. El valor del índice varía entre $-6,00$ y $+6,00$, estableciendo situación de sequía en un $36 \%$ de las situaciones, condiciones normales en un 30\%, y períodos húmedos en el $34 \%$ restante.

La escala de Palmer divide las situaciones de sequía en cinco niveles: sequía incipiente entre $-0,50$ y $-0,99$, sequía suave entre $-1,00$ y $-1,99$, sequía moderada entre $-2,00$ y $-2,99$, sequía grave entre $-3,00$ y $-3,99$, y sequía extrema para un valor igual o inferior a $-4,00$.

\section{5. Índice Normalizado de Precipitación (NPI, Normalized Precipitation Index)}

Creado por Garrido (1999), según el propio autor «incorpora el poder descriptivo del PDSI y las ventajas de la estandarización del SPI». Puede ser aplicado a diversas escalas temporales (mensual, estacional o anual), aunque el autor la pone en práctica mensualmente para la provincia de Murcia y la cuenca del Segura.

La escala del NPI estable situación de sequía en un $31 \%$ de las situaciones, condiciones normales en un $38 \%$, y períodos húmedos en el $31 \%$ restante. Por su parte, los períodos de sequía son de cuatro clases: sequía suave cuando el valor del índice se sitúa entre $-1,00$ y $-1,99$, sequía moderada entre $-2,00$ y $-2,99$, sequía severa entre $-3,00$ y $-3,99$, y sequía extrema para un valor igual o inferior a $-4,00$.

\section{6. Índice de Sequía Oferta-Demanda (SDDI, Supply-Demand Drought Index)}

Creado por Rind et al. (1990) con una concepción similar a la del PDSI, en comparación con éste se caracteriza por su sencillez, al prescindir de valores de recarga hídrica, escorrentía y pérdida de humedad en el suelo. La formulación es dSDDI=P-Ep-(P-Ep) clim, siendo P la precipitación, Ep la evapotranspiración potencial, y (P-Ep) clim el valor climatológico del índice en cada lugar o área. Puesto que el SDDI se calcula individualmente en cada lugar del planeta sin importar su tipología climática (por definición, el valor climatológico es igual a cero, por lo que la fórmula se reduce a $\mathrm{d}_{\mathrm{SDDI}}=\mathrm{P}-\mathrm{Ep}$ ), el índice carece de utilidad comparativa, y los resultados del mismo pueden ser más extremos en una región húmeda que en el ámbito desértico.

$\mathrm{Al}$ igual que el PDSI, este índice prevé un cálculo acumulativo mensual para establecer la evolución del período seco o húmedo. Partiendo del índice de anomalía de humedad $Z_{\mathrm{SDDI}}=\mathrm{d}_{\mathrm{sDDI}} / \mathrm{s}$, donde $\mathrm{s}$ es la desviación típica interanual de P-Ep para el mes en curso, se calcula el valor acumulado con la fórmula $Y_{(\mathrm{i})}=0,897 \cdot \mathrm{Y}_{(\mathrm{i}-1)}+Z_{(\mathrm{i})}$, donde 0,897 es un valor 
constante de mantenimiento del índice mes a mes, $\mathrm{Y}_{(\mathrm{i}-1)}$ es el valor acumulado del índice hasta el mes anterior, y $\mathrm{Z}_{(\mathrm{i})}$ es el valor del mes en curso.

La escala del SDDI, definida para el área continental de Estados Unidos, establece situación de sequía en un $36 \%$ de las situaciones, condiciones normales en un $28 \%$, y períodos húmedos en el $36 \%$ restante. En este caso, sólo se distinguen tres intervalos de sequía: sequía moderada entre $-0,90$ y $-3,29$, sequía fuerte entre $-3,30$ y $-6,29$, y sequía extrema para valores iguales o inferiores a $-6,30$.

\section{7. Índice Nacional de Lluvia (RI, National Rainfall Index)}

Fue creado por Gommes y Petrassi (1994), que lo aplicaron en África. Se calcula país por país, tomando la precipitación media anual del territorio según el peso de las medias a largo plazo de cada estación. El RI muestra una estrecha relación con la producción agrícola, puesto que al recaer el peso en la precipitación anual, las estaciones en áreas más húmedas de un país tienen una mayor influencia sobre el índice que las estaciones de áreas secas. Además, en África las estaciones lluviosas se localizan en regiones agrícolamente más productivas. Tiene de positivo el hecho de no quedar afectado por anomalías pluviométricas localizadas, la posibilidad de poder calcular el índice incluso sin disponer de datos de todas las estaciones, y la comparabilidad entre países. En el lado negativo, no permite una escala espacial de detalle (los resultados surgen en el ámbito nacional), y no es útil para detectar sequías meteorológicas o hidrológicas, sino sólo las agrícolas.

\section{8. Índice de sequedad $\mathrm{Si}$}

Creado por Ped (1975) para el antiguo territorio de la Unión Soviética, refleja la diferencia entre el cociente de las anomalías y la desviación típica de las anomalías de precipitación y temperatura: $\mathrm{Si}=\left(\Delta \mathrm{T} / \sigma_{\Delta \mathrm{T}}\right)-\left(\Delta \mathrm{Q} / \sigma_{\Delta \mathrm{Q}}\right)$, siendo $\Delta \mathrm{T}$ y $\Delta \mathrm{Q}$ las anomalías de temperatura y precipitación, y $\sigma_{\Delta \mathrm{T}}$ y $\sigma_{\Delta \mathrm{Q}}$ la desviación típica de las anomalías de temperatura y precipitación.

\subsection{Coeficiente hidrotérmico (HTC, Hydrotermal Coefficient)}

Creado por Selyanov (1928), también para la exURSS, se formula de la siguiente forma: $\mathrm{HTC}=\sum \mathrm{Q} / 0,1 \cdot \sum \mathrm{T}$, siendo $\mathrm{Q}$ la precipitación y $\mathrm{T}$ la temperatura superior a $10^{\circ} \mathrm{C}$ para un determinado período (habitualmente un mes o una estación vegetativa).

\subsection{0. Índice de Anomalía de Precipitación (RAI, Rainfall Anomaly Index)}

Creado por Van Rooy (1965), compara la precipitación de un lugar y momento determinados con la media de los diez valores extremos de anomalías positivas y negativas de precipitación. Es un índice poco conocido, sólo citado por Byun y Wilhite (1999), y aplicado de forma experimental en áreas muy concretas.

\subsection{Monitorización de Sequía (Drought Monitor)}

Más que un índice propiamente dicho, se trata de una síntesis de índices, una reelaboración de diversos indicadores de tipo meteorológico (PDSI o CMI de Palmer, y porcentaje 
de la precipitación media), edafológico (modelo de humedad en el suelo del Centro de Predicción Climática, y modelo de humedad en la capa superficial de suelo del USDA, o Departamento de Agricultura de Estados Unidos), hidrológico (percentil del caudal diario) y biológico (índice por satélite de salud de la vegetación), para la obtención de una clasificación de severidad de la sequía. Ha sido creado en un proyecto conjunto de diversas instituciones estadounidenses (Departamento de Agricultura, el Centro de Predicción Climática del Servicio Nacional de Meteorología, y el Centro Nacional de Mitigación de la Sequía de la Universidad de Nebraska), para contar con un mecanismo completo de identificación de áreas que padecen sequía en aquél país.

Tras el cálculo de los índices individuales, los resultados se cruzan en una tabla para definir cinco tipos de situaciones de sequía, cada una de las cuales lleva asociados una serie de impactos, principalmente relacionados con la actividad agrícola y el abastecimiento de agua (NDMC, 1999):

- D0 - Seco: Impactos menores, pero que causan preocupación.

- D1 - Sequía: Algunos daños a cultivos; alto riesgo de incendio; ríos, embalses y pozos con poca agua; escasez inminente de agua.

- D2 - Sequía severa: Pérdidas moderadas de cultivos y pastos; riesgo de incendio muy alto; restricciones de agua.

- D3 - Sequía extrema: Graves pérdidas de cultivos y pastos; riesgo extremo de incendio; restricciones de agua extensivas.

- D4 - Sequía excepcional: Pérdidas de cultivos y pastos excepcionales y extensivas; riesgo extremo de incendios a gran escala; escasez de agua en embalses, ríos y pozos, cansando restricciones y racionamientos extensivos.

En función de los criterios empleados para definir los intervalos de sequía, este índice es utilizado tanto para sequías meteorológicas, como hidrológicas y agrícolas.

\subsection{Precipitación Efectiva (EP, Effective Precipitation)}

Byun y Wilhite (1999) proponen, bajo el concepto de precipitación efectiva (sumatorio para un período determinado de la precipitación diaria modificado por una función de reducción dependiente en el tiempo), diversas opciones para calcular la duración y severidad de las sequías. Los autores defienden esta propuesta basándose en las deficiencias del resto de índices: falta de precisión en la detección del inicio, finalización e intensidad acumulada del período seco, falta de consideración de la incidencia temporal sobre la precipitación registrada de factores como la evapotranspiración o la escorrentía, e imposibilidad de realizar un seguimiento en tiempo real del evento seco, dado que la escala de trabajo es mensual (el índice propuesto funciona a escala diaria).

Para el cálculo de la precipitación efectiva (EP) se proponen tres fórmulas (los autores aceptan que puede haber muchas otras, y quizá mejores), cada una de las cuales introduce un factor de reducción temporal diferente: lineal suave, lineal fuerte y exponencial inversa. Posteriormente, se computa para cada día del calendario la precipitación efectiva media (MEP), la desviación de precipitación efectiva (DEP), o diferencia entre EP y MEP, y la precipitación efectiva estandarizada (SEP), o cociente entre DEP y una desviación típica de EP.

Obtenidos estos parámetros para cada día del año, la severidad de una sequía es cuantificada mediante diversos índices: 
- CNS (Consecutive Days of Negative SEP), o suma de días consecutivos con un valor negativo de precipitación efectiva estandarizada, mide la duración del déficit de precipitación.

- ANES (Accumulation of consecutive negative SEP), o sumatorio de los valores negativos de precipitación efectiva estandarizada, mide la acumulación del déficit pluviométrico durante todo el período seco (severidad).

- APD (Accumulated precipitation deficit), o sumatorio del déficit de precipitación durante todo el período seco. Se obtiene restando a la precipitación diaria el promedio de la precipitación de todo el período que ha registrado déficit pluviométrico, y sumando sucesivamente los valores diarios mientras dure el período seco.

- EDI (Effective Drought Index), o índice de sequía efectiva, proporciona el cociente entre la desviación de precipitación efectiva para todo el período seco y la desviación típica de la desviación de precipitación efectiva para todo el período seco: $\mathrm{DEP}_{\mathrm{j}} / \sigma \sigma \mathrm{DEP}_{\mathrm{j}}$.

- PNS (Percent normal as a second kind), o cociente entre el déficit acumulado de precipitación para todo el período seco y la precipitación media para todo el período seco: $\mathrm{APD}_{\mathrm{j}} / \mu_{\mathrm{j}}$.

El índice es todavía muy reciente para valorarlo adecuadamente, pero los resultados que los autores ofrecen para Nebraska (Estados Unidos) reflejan que permite llevar a cabo un seguimiento en tiempo real de la evolución de la sequía. Entre sus debilidades, cabe destacar la incógnita de su efectividad al aplicarlo en regiones con precipitación estacional, dado que durante la estación seca, con promedios mínimos o nulos de precipitación, un episodio aislado de lluvias podría dar por finalizada fácilmente la sequía, o dividirla artificialmente en dos o más períodos secos de corta duración, cuando en realidad el período seco presenta continuidad.

\subsection{3. Índice de Sequía (IS)}

Guerra y Almarza (1996) consideran determinantes para cuantificar las sequías dos factores, el déficit hídrico $\left(\mathrm{F}_{1}\right)$ y la persistencia $\left(\mathrm{F}_{2}\right)$, por lo que su índice viene definido por el producto de ambos: $I S=F_{1} \times F_{2}$. El déficit hídrico es formulado como el cociente entre la diferencia del valor medio de las precipitaciones de los últimos 12 meses y el valor medio mensual de toda la serie, y el coeficiente $\varepsilon\left(\mathrm{F}_{1}=\left[\mu_{1}-\mu\right] / \varepsilon\right)$, siendo $\varepsilon=\left\{\left[\sigma^{2}{ }_{1}+\sigma^{2}{ }_{2}\right] 12\right\}^{1 / 2}$. La persistencia es un factor de corrección que tiene en cuenta el número de meses de déficit hídrico $(\mathrm{n}): \mathrm{F}_{2}=[1+0,1 \cdot \mathrm{n}]$.

El índice ha sido aplicado por los autores en Bilbao y Madrid para caracterizar los episodios de sequía acaecidos a finales de los 80 y comienzos de los 90, pero adolece del establecimiento de algún marco de referencia que permita determinar la intensidad relativa del evento seco.

\section{Métodos de cuantificación de la sequía hidrológica}

\section{1. Índice Hidrológico de Sequía de Palmer (PHDI, Palmer Hydrologic Drought Index)}

El índice de sequía hidrológica de Palmer surge de introducir modificaciones al PDSI, destinadas a detectar las anomalías de humedad que afectan a la corriente de agua superfi- 
cial, la disponibilidad de agua en el suelo y el nivel de agua en lagos o embalses. El PHDI evoluciona muy lentamente de un mes a otro, de tal forma que no es útil para detectar la sequía meteorológica, ya que las reservas de agua no empiezan a resultar afectadas hasta que ésta se ha prolongado en el tiempo. De igual forma, los valores del PHDI permanecen negativos meses después de que el PDSI retorne a valores próximos a cero, dado el tiempo necesario de recarga de las reservas hídricas. En cualquier caso, los valores de PDSI y PHDI son casi equivalentes, manteniendo los mismos valores de referencia para definir las situaciones de sequía.

\section{2. Índice de Sequía por Humedad en el Suelo (SMDI, Surface Moisture Drought Index)}

Desarrollado por Hollinger et al. (1993), mide las anomalías anuales de humedad en el suelo, comparadas con el sumatorio del contenido diario de humedad en el suelo durante un año. Escasamente utilizado, sólo aparece citado en Byun y Wilhite (1999).

\section{3. Índice de Sequía - Demanda (RDI, Reclamation Drought Index)}

Desarrollado por Weghorst (1996), permite conocer el equilibrio entre oferta y demanda hídrica. En el cálculo se incluyen las variables precipitación, temperatura, espesor de nieve en el suelo, caudal, y reservas almacenadas. Su reciente creación hace que todavía sea un índice poco contrastado y de escasa aplicación.

\section{4. Índice de Sequía Keetch-Byram (KBDI, Keetch-Byram Drought Index)}

Este índice fue creado por el Servicio Forestal de EE.UU. (USDAFS, 1999), para medir específicamente la incidencia de la sequía en la capa superficial de suelo y humus (se basa en una capacidad de retención de agua de $20,32 \mathrm{~mm}$ ), siendo este factor el mejor indicador para establecer la probabilidad de ocurrencia de un incendio forestal. En el cálculo del índice, cuyo valor oscila entre 0 y 800 , se incluyen las variables temperatura máxima diaria, precipitación diaria, precipitación antecedente y precipitación anual. Los límites de intervalos del índice determinan el estado de la capa de suelo superficial y su contribución a la intensidad de los incendios forestales:

- 0-200: La humedad en el suelo y el combustible son elevados, por lo que no contribuyen demasiado a la intensidad del fuego. Típico del inicio de la estación de primavera.

- 200-400: Típico del final de la primavera, inicio de la estación de crecimiento. Las capas de humus y detritus se secan y empiezan a contribuir a la intensidad del fuego.

- 400-600: Típico del final de verano, inicio de otoño. Las capas de humus y detritus contribuyen activamente a la intensidad del fuego y se quemarán activamente.

- 600-800: A menudo asociado con las sequías más severas e incremento de ocurrencia de incendios forestales. Se pueden esperar incendios intensos en los que el combustible vivo también arderá activamente. 


\section{Métodos de cuantificación de la sequía agrícola}

\section{1. Índice $Z(Z I N X, Z$ Index)}

El índice Z mide la anomalía mensual de humedad en un área para un mes determinado. Se utiliza estrictamente para medir la intensidad de la sequía agrícola, dado que responde con rapidez a anomalías negativas de humedad de corta duración, y permite detectar aquellos picos de precipitación durante un período de sequía meteorológica o hídrica que favorecen una producción agrícola próxima a la de un año normal (en ese caso, el ZINX adquiere valor positivo, dando por finalizada la sequía agrícola). Karl (1986) señala que, para un seguimiento en tiempo real de las condiciones de sequía, el índice $\mathrm{Z}$ es más adecuado que el PDSI o el PHDI, ya que refleja fielmente las variaciones más inmediatas.

Los límites de intervalos del ZINX fueron establecidos por el NCDC (1989), de tal forma que un $28 \%$ de las situaciones son de sequía, un $44 \%$ se corresponde con condiciones normales, y el $28 \%$ restante son períodos húmedos. El índice distingue tres intervalos de sequía: sequía débil a moderada entre $-1,25$ y $-1,99$, sequía fuerte entre $-2,00$ y $-2,74$, y sequía extrema para un valores iguales o inferiores a $-2,75$.

\section{2. Índice de Humedad del Cultivo (CMI, Crop Moisture Index)}

El prolífico Palmer (1968) creó el CMI para medir las variaciones semanales de disponibilidad de humedad para el cultivo. Utilizado estrictamente para medir la intensidad de la sequía agrícola, se basa en datos semanales de temperatura media y precipitación total, y en el valor del CMI de la semana anterior, por lo que responde rápidamente a variaciones de estas variables y no es útil para medir la sequía meteorológica. Una muestra de su limitación es que el CMI se ajusta a cero en cada época de crecimiento del cultivo, para evitar la acumulación de anomalías fuera de este período. Como el resto de índices creados por Palmer, éste es utilizado casi exclusivamente en Estados Unidos, y ha sido adoptado por el Departamento de Agricultura de este país, que lo calcula y publica semanalmente en el Weekly Weather and Crop Bulletin (Wilhite y Glantz, 1985).

\section{3. Índice de Sequía Específico de Cultivo (CSDI, Crop-Specific Drought Index)}

Índice creado por Meyer et al. (1993), muestra el cociente entre el sumatorio de la evapotranspiración calculada para el período de crecimiento de determinados cultivos, y la evapotranspiración media de ese mismo período. Este método, al ser aplicado para cultivos específicos, recibe las siguientes denominaciones: Índice de Sequía del Maíz (CDI, Corn Drought Index), desarrollado en Meyer et al. (1993), e Índice de Sequía de la Soja (SDI, Soybean Drought Index), descrito en Meyer y Hubbard (1995).

\section{4. Índice de Aporte de Agua Superficial (SWSI, Surface Water Supply Index)}

Fue desarrollado por Shafer y Dezman (1982) para permitir una mejor aplicación del PDSI en el estado de Colorado (EE.UU.), donde el índice de Palmer no se mostraba efectivo. De hecho, el PDSI presenta problemas en todas aquellas regiones de topografía variada, donde los suelos no son homogéneos o hay precipitación nival. El SWSI requiere datos de cubierta nivosa, corriente superficial de agua, precipitación y reserva hídrica, pon- 
derándose cada variable según su contribución al agua superficial existente en cada cuenca. Por último, los componentes se suman para obtener el valor final de SWSI, que oscila entre $+4,2$ (extremadamente húmedo) y $-4,2$ (sequía extrema), y se divide en intervalos como los del índice de Palmer. Las variables utilizadas determinan que el índice sea dependiente de la estación, por lo que los datos de cubierta nivosa sólo se utilizan en los meses de invierno, y los de corriente superficial de agua en los meses de verano. El método de cálculo escogido obliga a introducir constantes cambios en la formulación del índice, y su aplicabilidad es bastante limitada, de tal forma que actualmente sólo se utiliza en estados montañosos y de invierno duro de Estados Unidos: Colorado, Oregón, Montana, Idaho y Utah.

\subsection{Lluvias dependientes (DR, Dependable Rains) / Tasa de fiabilidad (TF)}

Este índice define las lluvias dependientes como aquellas que se producen en tres de cada cuatro años (Hargreaves, 1974), o en cuatro de cada cinco años (Dancette y Hall, 1979; Le Houerou et al., 1993). Se trata tan sólo de un valor de referencia (equivalente a los percentiles 75 u 80, según el caso), a partir del cual la producción agrícola queda comprometida y que sirve como límite singular por debajo del cual se produce una sequía agrícola, mientras que por encima indica que las precipitaciones son fiables para el ciclo económico. El valor de DR varía en África entre el 40-50\% de la media anual cerca del Sahara, y el $80 \%$ de la media en las zonas con precipitación anual de 700-800 mm. Una variación de este índice es la tasa de fiabilidad, que señala la relación entre las lluvias dependientes y la precipitación media.

\section{6. Índice DM de sequedad-humedad (DM Index, Dry Conditions and Excessive Moisture Index)}

Creado por Meshcherskaya y Blazhevich (1997) para el área productora de grano en la exURSS, se trata de un índice espacial basado en dos fórmulas, una para calcular las condiciones de sequedad (Índice D) y otro para el exceso de humedad (Índice M). Puesto que el área productora de grano en la exURSS se caracteriza por tener una correlación negativa entre precipitación y temperatura, los autores establecen los umbrales a partir de los cuales un determinado período es seco (precipitación inferior a un $80 \%$ de la media y anomalía positiva de temperatura superior a $1^{\circ} \mathrm{C}$ ) o húmedo (precipitación superior a un $120 \%$ de la media y anomalía negativa de temperatura superior a $1^{\circ} \mathrm{C}$ ).

Para que el período considerado en una determinada estación sea calificado como seco o húmedo deben cumplirse simultáneamente las dos condiciones, por lo que también cabe la posibilidad de una situación neutra. El umbral de precipitación fue escogido por los autores tras detectar que, para el período 1891-1975, un tercio de los valores era inferior al $80 \%$ de la media, otro tercio era superior a un $120 \%$ de la media, y el tercio restante se situaba entre el 80 y el $120 \%$ de la media; los umbrales de temperatura limitan un intervalo de normalidad $\left( \pm 1^{\circ} \mathrm{C}\right)$ donde se sitúan un $40 \%$ de los valores, mientras que el $60 \%$ restante se reparte entre los dos extremos superior e inferior.

Una vez establecida la situación de cada estación, los Índices D y M son sometidos a una media areal, obteniendo como resultado el porcentaje de territorio afectado por condiciones de sequía o humedad para el período considerado (los autores lo aplicaron para el período mayo-junio que es el que más influye en el crecimiento del cereal en el granero exsoviético). Finalmente, el Índice DM se obtiene por la diferencia entre los dos índices: si el valor es 
positivo, el área padece sequía, si es negativo, predominan las condiciones de humedad. En función de las características climáticas de la región a la que se aplique, los autores proponen diferentes umbrales de referencia para clasificar los períodos en tres intervalos (seco, normal y húmedo), cada uno de los cuales se reparten el 33\% de las ocurrencias.

\section{Métodos de cuantificación de la sequía socioeconómica: ¿una misión imposible o un problema no resuelto?}

Existe, como se ha podido comprobar en anteriores apartados, una amplia variedad de fórmulas con las que delimitar la duración y definir la magnitud de los episodios de sequía meteorológica, hidrológica e incluso agrícola. Cada una de ellas presenta aspectos positivos y negativos que limitan o potencian su utilización en determinadas regiones planetarias. Sin embargo, ninguno de estos índices es válido para detectar las características de las sequías socioeconómicas.

Actualmente, para evaluar y caracterizar la sequía socioeconómica, la única referencia cuantitativa existente son las relaciones de pérdidas económicas derivadas de la escasez hídrica, o el número de personas afectadas por restricciones de abastecimiento de agua y, en casos extremos, el recuento de fallecidos.

Admitiendo la utilidad de esta información, que puede servir para caracterizar los sectores económicos y los grupos sociales más castigados por la sequía, existe una evidente carencia metodológica en la determinación de la vulnerabilidad de la estructura socioeconómica de una región a los episodios de indigencia pluviométrica.

El componente vulnerabilidad determina el grado de sensibilidad de un espacio geográfico, la población que lo habita, las actividades económicas que desarrollan, y el tejido social que lo caracteriza, a la escasez de precipitaciones. Son vulnerables los grupos humanos, actividades o territorios que afrontan el riesgo de efectos negativos extremos como resultado de eventos climáticos que sobrepasan las adaptaciones en curso (Stern y Easterling, 1999), de tal forma que la vulnerabilidad se halla estrechamente relacionada con la estructura social y el desarrollo económico, y está sometida a las variaciones y cambios que experimenten el tejido social y la estructura económica.

Por tanto, dos regiones distintas con idénticas características climáticas (promedio anual de temperatura y precipitación, variabilidad interanual, estacionalidad, evapotranspiración, etc.), padecerán de forma muy distinta los efectos de una anomalía de precipitación idéntica en ambas zonas, en función de su grado de desarrollo socioeconómico o, en otras palabras, de desarrollo humano. Entiendo por desarrollo humano el concepto detallado por el PNUD (1990), cuya definición supera la mera acumulación de riqueza imperante en la clásica concepción económica de desarrollo, y recoge elementos de bienestar social: «el propósito del desarrollo es la creación de un entorno capaz de permitir que la población disfrute una vida larga, saludable y creativa».

¿Cómo se resuelve esta disparidad mediante los índices climáticos disponibles? La solución más común ha sido la aplicación de métodos diferentes y/o umbrales de intensidad distintos en función del nivel de desarrollo económico de la región estudiada. En áreas desarrolladas, con una sociedad y una economía menos vulnerables a los extremos climáticos, el grado de amplitud de precipitación alrededor del valor intermedio que podrá ser soportado sin alteraciones destacables en su funcionamiento es amplio. Por tanto, el área comprendida bajo la denominación de 'normal' puede extenderse en la distribución frecuencial hasta cerca de un $50 \%$ de los valores. 
Tal es el caso de la estructura de McKee et al. (1993) con el SPI, donde el método estadístico y los umbrales escogidos hacen que un 55\% de las observaciones sean calificadas como de precipitación normal (véase la figura $\mathrm{n}^{\mathrm{o}} 3$ ). Sin embargo, si aplicamos el mismo método a áreas menos desarrolladas y más desestructuradas, se producirán episodios de sequía socioeconómica que no habrán sido detectados por el SPI, al quedar encuadrados en la designación 'normal'. En regiones menos desarrolladas e incapaces de hacer frente a las anomalías pluviométricas, la mayor vulnerabilidad a los episodios extremos provoca que el índice no sea operativo. Una solución, no exenta de subjetividad, sería reducir el área comprendida bajo el intervalo de precipitación normal, disminuyendo los umbrales de desviación desde $\pm 0,75$, hasta $\pm 0,50$ o $\pm 0,25$, según la vulnerabilidad de la región afectada.

En el caso de los cuantiles (deciles según la definición de intensidades establecida por Gibbs y Maher, 1967), la clasificación es especialmente adecuada para áreas menos desarrolladas, al reducir el espacio de precipitación 'normal' a un $20 \%$ de las observaciones. En este caso, el problema es el inverso, puesto que al aplicar el mismo método a regiones desarrolladas quedarán identificados episodios secos que no han tenido reflejo alguno en la sociedad y/o la economía de esa región. Nuevamente, se pueden variar los umbrales para adaptarlos a la vulnerabilidad del área de estudio, situando la denominación de normal, por ejemplo, entre los percentiles 30 y 70 , de forma que un $40 \%$ de los valores queden en el

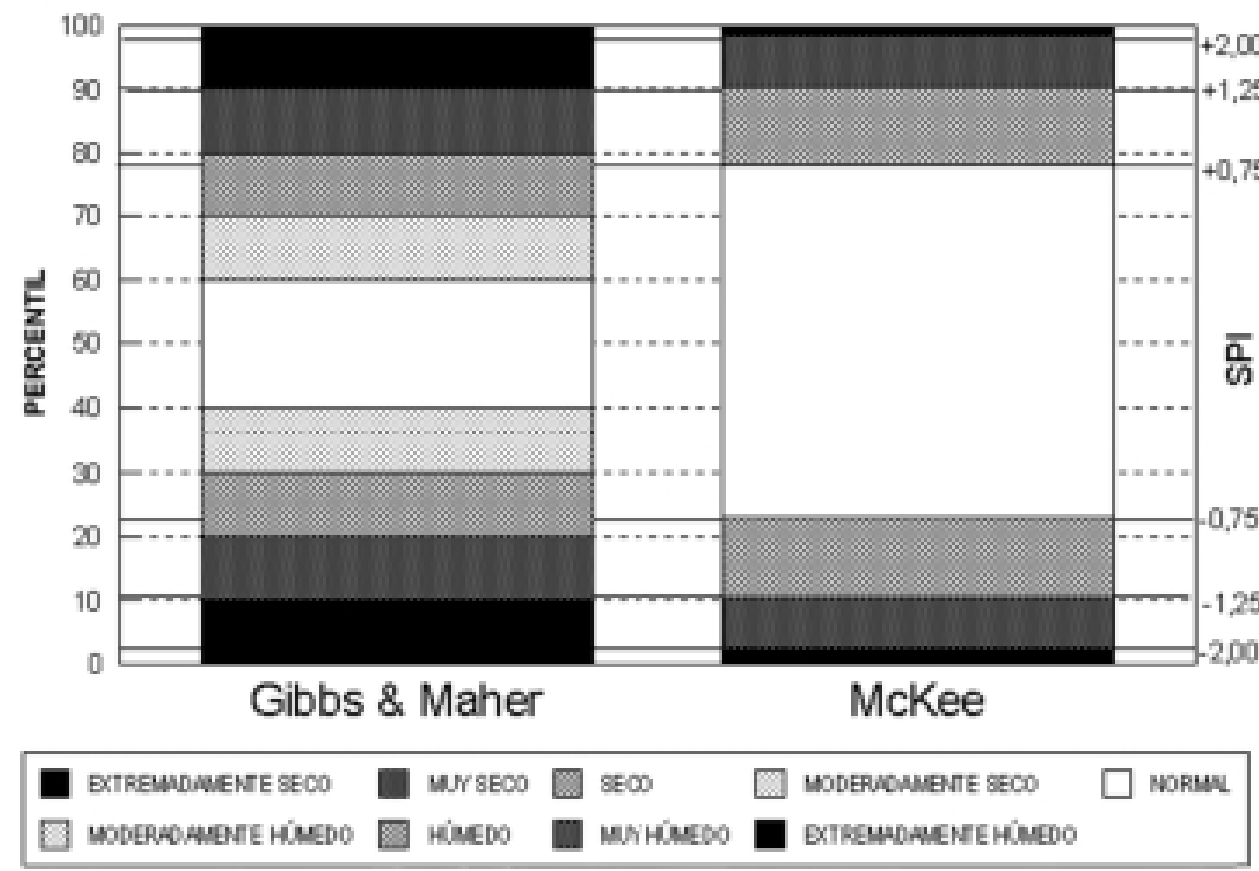

FUENTE: Gibbs y Maher (1967) para la escala de deciles, y McKee et al. (1993), para la del índice de precipitación estandarizada.

Figura 3. Comparación de los umbrales que determinan la intensidad de la sequía según el método de los deciles y el índice de precipitación estandarizada. 
intervalo intermedio. En cualquier caso, el proceso de adaptación nuevamente tendrá un carácter subjetivo al carecer de una herramienta objetiva de cuantificación.

Si cambiamos la escala espacial por la temporal el problema es idéntico, puesto que la caracterización de la intensidad de la sequía en una misma área a lo largo del tiempo se halla sometida a diferentes contextos de vulnerabilidad a la indigencia pluviométrica, en función de la propia evolución social y económica de esa región. Así, dos episodios secos que se produzcan en el mismo espacio geográfico en dos períodos distintos, y cuya intensidad sea igual al aplicar el mismo método estadístico, serán percibidos de forma muy diferente en función de la vulnerabilidad, y ésta está en función de parámetros socieconómicos. Para resolver esta incoherencia, podremos cambiar de método (el de cuantiles durante la fase de menor desarrollo, y el SPI en la de mayor desarrollo), o modificar los umbrales de intensidad progresivamente, pero ¿cuándo debemos cambiar de método?, y ¿en qué cuantía han de variar los umbrales para adaptarse a la realidad socioeconómica del momento?

La dificultad resultante es clara: ¿cómo determinar el grado de vulnerabilidad de una sociedad a los eventos extremos? y, lo que es más complejo, ¿cómo trasladar esa vulnerabilidad al índice de forma que los umbrales queden establecidos con un método objetivo? Ante la dualidad del problema, la respuesta parece residir en la combinación de indicadores climáticos y socioeconómicos, adaptando en el tiempo los umbrales de referencia en función de la variación que registren las condiciones de respuesta de la sociedad y la economía de una región a los episodios de sequía.

\section{Bibliografía}

ALLEY, W.M. (1984): 'The Palmer Drought Severity Index: limitations and assumptions'. Journal of Climate and Applied Meteorology, 23: 1100-1109.

BHALME, H.N. y MOOLEY, D.A. (1980): 'Large-scale drought/floods and monsoon circulation'. Monthly Weather Review, 108: 1179-1211.

BOWLES, D.S., HUGHES, T.C., JAMES, W.R., JENSEN, D.T. y HAWS, F.W. (1980): Vulnerability of water supply systems to droughts (UWRL/P-80/08) Water Research Laboratory, College of Engineering (Utah State University). Logan, Utah. 67 pp.

BURTON, I., KATES, R.W. y WHITE, G.F. (1978): The environment as hazard. Oxford University Press. New York. 240 pp.

BYUN, H.-R. y WILHITE, D.A. (1999): 'Objective quantification of drought severity and duration'. Journal of Climate, 12: 2747-2756.

DANCETTE, C. y HALL, A.E. (1979): 'Agroclimatology applied to water management in the Sudanian and Sahelian zones of Africa'. En: HALL, A.E., CANNELL, G.H. y LAWTON, H.W. (Eds.): Agriculture in semiarid environments. Ecological Studies, vol. 34. Springer Verlag. Heidelberg.

DHAR, O.N., RAKHECHA, P.R. y KULKARNI, A.K. (1979): 'Rainfall study of severe drought years of India'. Symposium on Hydrological Aspects of Droughts, Nueva Delhi (India). Comité Nacional Indio para el Programa Hidrológico Internacional.

DRACUP, J.A., LEE, K.S., PAULSON JR., E.G. (1980): 'On the statistical characteristics of drought events'. Water Resources Research, 16 (2): 289-296.

ENTEKHABI, D., RODRÍGUEZ-ITURBE, I. y BRAS, R.L. (1992): 'Variability in largescale water balance with land surface-atmosphere interaction'. Journal of Climate, 5: 798-813. 
GARRIDO, R. (1999): 'A drought watch system for southest Spain'. Drought Network News, 11 (2): 3-7.

GIBBS, W.J. y MAHER, J.V. (1967): 'Rainfall deciles as drought indicators'. Bureau of Meteorology Bulletin, 48. Commonwealth of Australia, Melbourne.

GOMMES, R. y PETRASSI, F. (1994): Rainfall variability and drought in Sub-Saharan Africa since 1960. Agrometeorology Series Working Paper N. 9. Food and Agriculture Organization, Roma. 100 pp.

GOUDIE, A. (Ed.) (1985): Encyclopaedic dictionary of physical geography. Blackwell. Oxford. $528 \mathrm{pp}$.

GUERRA SIERRA, J. y ALMARZA MATA, C. (1996): ‘Análisis del último período seco en Madrid por medio de un nuevo índice estadístico'. En MARZOL, M.V., DORTA, P. y VALLADARES, P. (Eds.): Clima y agua: la gestión de un recurso climático. Actas de la III Reunión Nacional de Climatología. Universidad de La Laguna - Asociación de Geógrafos Españoles. Santa Cruz de Tenerife. (pp. 291-298).

HAVENS, A.V. (1954): 'Drought and agriculture'. Weatherwise, 7: 51-55.

HARGREAVES, G.H. (1974): Precipitation dependability and potential for agricultural production in Northeast Brazil. EMBRAPA \& Utah State University. Logan, Utah. 123 pp.

HOLLINGER, S.E., ISARD, S.A. y WELFORD, M.R. (1993): 'A new soil moisture drought index for predicting crop yields'. En: Preprints, Eighth Conference on Applied Climatology. American Meteorological Society. Anaheim, California. (pp. 187-190).

HUDSON, H.E. y HAZEN, R. (1964): 'Drought and low stream flow'. En: CHOW, V.T. (Ed.): Handbook of applied hydrology. McGraw-Hill. New York. Capítulo 18.

KARL, T.R. (1986): 'The Sensitivity of the Palmer Drought Severity Indez and Palmer's Z-Index to their Calibration Coefficients Including Potential Evapotranspiration'. Journal of Climate and Applied Meteorology, 25: 77-86.

KRISHNAN, A. (1979): 'Definition of droughts and factors relevant to specification of agricultural and hydrological droughts'. Symposium on Hydrological Aspects of Droughts, Nueva Delhi (India). Comité Nacional Indio para el Programa Hidrológico Internacional.

KULIK, M.S. (1962): 'Agroclimatic indices of drought'. En: DAVIDAYA, F.F. y KULIK, M.S. (Eds.): Compendium of abridged reports to the Second Session of CagM (WMO). Hydrometeorological Publishing. Moscú. (pp. 75-81).

LE HOUÉROU, H.N., POPOV, G.F. y SEE, L. (1993): Agro-bioclimatic classification of Africa. Agrometeorology Series Working Paper N. 6. Food and Agriculture Organization, Roma.

LINSLEY JR., R.K., KOHLER, M.A. y PAULHUS, J.C.H. (1975): Hydrology for engineers ( $2^{\mathrm{a}}$ edición). McGraw Hill. New York.

MARTÍN-VIDE, J. (1998): 'Cambios en el sistema climático: escalas, métodos y efectos (desertificación)'. En: Anales del Seminario Internacional sobre Inserción del Semiárido Latinoamericano en el Proceso de Globalización de la Economía Mundial. IPA UNIFACS. Salvador de Bahia. (pp. 515-528).

McKEE, T.B., DOEKSEN, N.J. y KLEIST, J. (1993): 'The relationship of drought frequency and duration to time scales'. Preprints 8th Conference on Applied Climatology, 17-22 enero, Anaheim (California, EE.UU.), pp. 179-184.

MESHCHERSKAYA, A.V. y BLAZHEVICH, V.G. (1997): 'The drought and excessive moisture indices in a historical perspective in the principal grain-producing regions of the former Soviet Union'. Journal of Climate, 10: 2670-2682. 
MEYER, S.J. y HUBBARD, K.G. (1995): 'Extending the crop-specific drought index to soybean'. En: Preprints, Ninth Conference on Applied Climatology. American Meteorological Society. Dallas, Texas. (pp. 233-236).

MEYER, S.J., HUBBARD, K.G. y WILHITE, D.A. (1993): 'The development of a cropspecific drought index for corn. Part I: Model development and validation'. Agronomy Journal, 85: 388-395.

NCDC (1989): Time Bias Corrected Divisional Temperature-Precipitation-Drought Index. National Climatic Data Center. Asheville, NC.

NDMC (1995): 'Understanding and Defining Drought'. [En línea]. Página web, Lincoln (Nebraska): National Drought Mitigation Center - University of Nebraska. <http://enso.unl.edu/ndmc/enigma/def2.htm>. Actualización 15 de noviembre de 1995. [Consulta, 27 de septiembre de 1996].

NDMC (1996): 'Drought Indices'. [En línea]. Página web, Lincoln (Nebraska): National Drought Mitigation Center - University of Nebraska. <http://enso.unl.edu/ndmc/enigma/indices.htm>. Actualización 3 de julio de 1996. [Consulta, 27 de septiembre de 1996].

NDMC (1999): 'Drought Monitor: State-of-the-Art Blend of Science and Subjectivity'. [En línea]. Página web, Lincoln (Nebraska): National Drought Mitigation Center - University of Nebraska. <http://enso.unl.edu/monitor/archive/99/classify.htm>. Actualización semanal. [Consulta, 18 de octubre de 1999].

OLCINA, J. (1994): Riesgos climáticos en la Península Ibérica. Acción Divulgativa (Col. Libros Penthalon). Madrid. $440 \mathrm{pp}$.

PALMER, W.C. (1965): Meteorological drought. Research Paper N. 45, U.S. Department of Commerce Weather Bureau, Washington DC. 58 pp.

PALMER, W.C. (1968): 'Keeping track of crop moisture conditions nationwide: the new Crop Moisture Index'. Weatherwise, 21: 156-161.

PED, D.A. (1975): 'Indicadores de sequías y condiciones de humedad' (original en ruso). Anales Centro Hidrometeorológico de la URSS, 156: 19-39.

PITA LÓPEZ, M M F. (1995): Las sequías: análisis y tratamiento. Consejería de Medio Ambiente. Junta de Andalucía. Sevilla. 56 pp.

PNUD (1990): Human Development Report 1990: Concept and Measurement of Human Development. Programa de Naciones Unidas para el Desarrollo. Oxford University Press. New York.

RAMDAS, D.A. (1960): Crops and weather in India. ICAR. Nueva Delhi (India).

RASMUSSEN, E.M. (1987): 'Global climate change and variability: effects on drought and desertification in Africa'. En: GLANTZ, M. (Ed.): Drought and hunger in Africa. Cambridge University Press.

RIND, D., GOLDBERG, R., HANSEN, J., ROSENZWEIG, C. y RUEDY, R. (1990): 'Potential evapotranspiration and the likelihood of future drought'. Journal of Geophysical Research, 95: 9983-10004.

RUSSELL, C.S., AREY, D.G. y KATES, R.W. (1970): Drought and water supply. Johns Hopkins University Press. Baltimore (EE.UU.). 232 pp.

SELYANINOV, G.T. (1928): 'Variación del clima agrícola' (original en ruso). Anales Meteorología Agrícola, 20: 165-177.

SHAFER, B.A. y DEZMAN, L.E. (1982): 'Development of a Surface Water Supply Index (SWSI) to assess the severity of drought conditions in snowpack runoff areas'. Proceedings of the Western Snow Conference, pp. 164-175. 
SMITH, D.I., HUTCHINSON, M.F. y McARTHUR, R.J. (1993): 'Australian climatic and agricultural drought: payments and policy'. Drought Network News, 5 (3): 11-12.

SOULE, P.T. (1992): 'Spatial patterns of drought frequency and duration in the contiguous USA based on multiple drought event definitions'. International Journal of Climatology, 12: 11-24.

STERN, P.C. y EASTERLING, W.E. (Eds.) (1999): Making climate forecasts matter. National Research Council. National Academy Press. Washington DC. 176 pp.

SUBRAHMANYAM, V.P. (1967): Incidence and spread of continental drought. (WMO/IHD Report $\mathrm{n}^{\circ}$ 2). Organización Meteorológica Mundial. Ginebra.

SUDENE (1981): As secas do Nordeste. Uma abordagem histórica de causas e efeitos. Superintendéncia do Desenvolvimento do Nordeste. Recife. 82 pp + 38 mapas.

SUDENE (1999): 'O fenômeno das secas'. [En línea]. Página web, Recife: Superintendencia do Desenvolvimento do Nordeste. <http://www.sudene.gov.br/nordeste/ Seca.html>. [Consulta, 14 de enero de 2000].

TOUPET, Ch.-H. (1989): 'Comparaison des sécheresses historiques et de la sécheresse actuelle au Sahel. Essai de définition de la sécheresse et de l'aridification'. En: BRET, B. (Ed.): Les hommes facent aux secheresses. Paris. IHEAL. (pp. 77-83).

VAN ROOY, M.P. (1965): 'A rainfall anomaly index independent of time and space'. Notos, 14, p. 43.

USDAFS (1999): 'Wildland Fire Assessment System: Drought maps'. [En línea]. Página web, Missoula (Montana): United States Department of Agriculture Forest Service, Rocky Mountain Research Station. <http://www.fs.fed.us/land/wfas/>. [Consulta, 25 de noviembre de 1999].

WEGHORST, K.M. (1996): The reclamation drought index: guidelines and practical applications. Bureau of Reclamation. Denver, Colorado. 6 pp.

WILHITE, D.A. y GLANTZ, M.H. (1985): 'Understanding the drought phenomenon: the role of definitions'. Water International, vol. 10: 111-120. 